\title{
Risk stratification score to determine long-term outcomes based on post-operative complications following cardiac surgery - need of the hour?
}

\author{
Siddharth Pahwa ${ }^{1}$ \\ ${ }^{1}$ Mayo Clinic
}

March 22, 2021

\begin{abstract}
Risk models were developed to provide clinicians and hospitals with a tool to evaluate risk-adjusted outcomes and to guide quality improvement. The Society of Thoracic Surgeons (STS) Predicted Risk of Mortality (PROM) is the most commonly used risk algorithm, others being the EuroSCORE logistic and additive algorithm and the Ambler Risk Score. These models utilize pre-operative patient characteristics to predict operative risk and early outcomes. Although a great deal of effort has gone into models to predict short-term patient outcomes after common cardiac operations, there has been relatively little effort to develop a statistical algorithm to predict long-term outcomes. Moreover, no risk model takes into account early post-operative complications to construct an algorithm to predict long-term outcomes. The formulation of a risk stratification score based on post-operative complications following common cardiac surgical procedures may be used to estimate the likelihood of longterm survival for individual complications, as well as various permutations and combinations of complications. This may have profound implications in devising strategies to prevent the most devastating combination of complications. Also, this may assist in informing patients and families of the predicted survival after a particular complication or a combination of complications. As Dokollari et all pointed out, there is impetus towards the direction of formulating a risk stratification score, and this may indeed be the need of the hour.
\end{abstract}

Risk stratification score to determine long-term outcomes based on post-operative complications following cardiac surgery - need of the hour?

Siddharth Pahwa, MD ${ }^{1}$

1 - Department of Cardiovascular Surgery and Thoracic Surgery, University of Louisville, KY

No sources of funding and conflicts of interest to disclose.

Corresponding author:

Siddharth Pahwa, MD

Department of Cardiovascular and Thoracic Surgery

University of Louisville, KY

Phone - 502-588-7600

Email - siddharth.pahwa@uoflhealth.org

Word count - 499 
Risk models were developed to provide clinicians and hospitals with a tool to evaluate risk-adjusted outcomes and to guide quality improvement. The Society of Thoracic Surgeons (STS) Predicted Risk of Mortality (PROM) is the most commonly used risk algorithm, others being the EuroSCORE logistic and additive algorithm and the Ambler Risk Score. These models utilize pre-operative patient characteristics to predict operative risk and early outcomes. Although a great deal of effort has gone into models to predict shortterm patient outcomes after common cardiac operations, there has been relatively little effort to develop a statistical algorithm to predict long-term outcomes. Moreover, no risk model takes into account early post-operative complications to construct an algorithm to predict long-term outcomes.

Dokollari and colleagues in their recent invited commentary rightly pointed out that there is a need for a clinical risk prediction model for post-operative complications. ${ }^{1}$ Recently published data by Seese and colleagues reported the impact of the STS-defined major morbidities (prolonged ventilation, deep sternal wound infection, renal failure, re-operation and stroke) on long-term outcomes. ${ }^{2}$ Pahwa and colleagues further reported the impact of seventeen common post-operative complications and analyzed their impact on long-term survival. ${ }^{3}$ The effect of multiple complications and various combinations of complications were also investigated and, understandably, the greater the number of complications, the worse was the survival. ${ }^{2,} 3$

The formulation of a risk stratification score based on post-operative complications following common cardiac surgical procedures may be used to estimate the likelihood of long-term survival for individual complications, as well as various permutations and combinations of complications. This may have profound implications in devising strategies to prevent the most devastating combination of complications. Also, this may assist in informing patients and families of the predicted survival after a particular complication or a combination of complications. As Dokollari et all pointed out, there is impetus towards the direction of formulating a risk stratification score, and this may indeed be the need of the hour. ${ }^{1}$

\section{References}

1. Dokollari A, Cameli M, Kalra DK, Gelsomino S, Bisleri G, Bonacchi M. Success in long-term outcome after cardiac surgery: Rise from an uncomplicated immediate postoperative course. J Card Surg. 2021 Mar 9. doi: $10.1111 /$ jocs.15467.

2. Seese L, Sultan I, Gleason TG, Navid F, Wang Y, Thoma F, Kilic A. The Impact of Major Postoperative Complications on Long-Term Survival After Cardiac Surgery. Ann Thorac Surg. 2020 Jul;110(1):128-135. doi: 10.1016/j.athoracsur.2019.09.100.

3. Pahwa S, Bernabei A, Schaff H, Stulak J, Greason K, Pochettino A, Daly R, Dearani J, Bagameri G, King K, Viehman J, Crestanello J. Impact of postoperative complications after cardiac surgery on long-term survival. J Card Surg. 2021 Mar 9. doi: 10.1111/jocs.15471. 